\title{
Quantum dot polarized light sources
}

This article has been downloaded from IOPscience. Please scroll down to see the full text article.

2011 Semicond. Sci. Technol. 26014002

(http://iopscience.iop.org/0268-1242/26/1/014002)

View the table of contents for this issue, or go to the journal homepage for more

Download details:

IP Address: 141.211.173.82

The article was downloaded on 06/04/2012 at 16:59

Please note that terms and conditions apply. 


\title{
Quantum dot polarized light sources
}

\author{
Pallab Bhattacharya ${ }^{1}$, Debashish Basu ${ }^{1}$, Ayan Das ${ }^{1}$ and Dipankar Saha ${ }^{2}$ \\ ${ }^{1}$ Solid State Electronics Laboratory, Department of Electrical Engineering and Computer Science, \\ University of Michigan, Ann Arbor, MI 48109, USA \\ ${ }^{2}$ Center of Excellence in Nanoelectronics, Department of Electrical Engineering, Indian Institute of \\ Technology Bombay, Mumbai 400 076, India
}

Received 25 May 2010, in final form 7 August 2010

Published 15 November 2010

Online at stacks.iop.org/SST/26/014002

\begin{abstract}
The design, operation and performance of quantum dot spin-polarized vertical cavity surface emitting lasers (VCSELs) and single-photon sources are described and discussed. The effects of spin-induced gain anisotropy on output polarization and threshold current reduction have been studied along with the high-frequency response in a spin-polarized VCSEL. While the output circular polarization in a VCSEL follows the out-of-plane magnetization characteristics of the ferromagnetic spin injector, the output polarization of the spin-polarized single-photon source shows a switching behavior which is explained by invoking the exciton fine structure in the quantum dots and the effects of electron-hole exchange splitting due to in-plane quantum dot rotational asymmetry.
\end{abstract}

(Some figures in this article are in colour only in the electronic version)

\section{Introduction}

Polarized light sources and in particular lasers and singlephoton sources with polarization control and switching would be useful for a host of applications including reconfigurable optical interconnects, cryptographic optical communications, optical switching and the study of vitamins, fibrous proteins, optically active biological structures, asymmetric photochemical synthesis and pharmacological properties of drugs [1]. The output circular polarization of semiconductor lasers is determined by the polarization properties of the active region and optical cavity [2]. In a vertical cavity surface emitting laser (VCSEL), polarization control and stability are obtained by intentionally introducing cavity and/or mirror asymmetry [3]. In a single-photon source, the polarization of each individual photon is uncontrolled and random and that of an ensemble integrated over some length of time would be an equal number of left- and right-circularly polarized photons. Therefore for polarization-encoded quantum cryptography, at least half of the photons are lost. With some kind of polarization control, the information flow can be enhanced [4]. In this context, techniques such as enhanced emission into a specific polarization mode of a high-Q cavity [5], or the introduction of asymmetry in the cavity have been investigated [6]. An elegant technique to control the polarization of the emitted photons is to inject spin-polarized carriers into the active (gain) region. The quantum selection rules for the conservation of angular momentum directly relate the spin orientation of the recombining carriers to the polarization of the emitted photons. Based on this principle, electrically injected spin light-emitting diodes (spin LEDs) [7] and spin lasers [8] have been demonstrated. A common feature in all these devices is that the output circular polarization follows the magnetization of the spin injecting contact, which confirms that the observed polarization of the light output is related to spin injection, and not spurious and uncorrelated effects. More recently, we have reported the output polarization characteristics of an electrically injected non-classical singlephoton source where spin-polarized electrons are injected to recombine with holes [9]. It is seen that in this device the output polarization does not follow the magnetization of the spin injector.

For high-temperature operation of spin-polarized lasers, it is advantageous to have self-organized InAs/GaAs quantum dots as the gain media since the discrete density of states and spatial localization of carriers in the dots inhibit the scattering processes which reduce the spin relaxation time. Similarly, quantum dots can serve as sources of single photons, without the necessity of Coulomb blockade in the operation of the device. From the carrier dynamics in these dots it is apparent that in addition to serving as a source of single photons, they can also generate entangled photons or an exciton-biexciton cascade [10]. The quantum dot emitter is usually embedded in a resonant microcavity, whereby its emission properties are 
modified by the Purcell effect [11]. Being an integral part of a semiconductor-based heterostructure system, quantum dots are amenable to the fabrication of practical electrically injected devices. Finally, the emission wavelength of the quantum dots can be tuned over a large range, from ultraviolet to $\sim 2 \mu \mathrm{m}$, and therefore the important wavelengths for long-distance optical communication are accessible. The first spin-lasers to be demonstrated, including operation at room temperature, were optically pumped [12]. However, electrically pumped devices are more practical; although, it relies on efficient spin injection of polarized carriers from a suitable ferromagnetic contact and subsequent transport of these carriers to the active region. In this paper, we will review recent progress made in the realization and characterization of electrically pumped spin-polarized VCSELs and single-photon sources.

\section{Basic principles}

Polarized light sources involve radiative recombination of spin-polarized carriers, which transfer their angular momentum to photons, generating circularly polarized light. The spin of \pm 1 which is carried away by the photon necessitates that an electron with spin $+1 / 2$ can only recombine with a hole of spin $-1 / 2$ or $+3 / 2$. Similarly, an electron with spin $-1 / 2$ in the conduction band (CB) can recombine with light holes $(\mathrm{LH})$ of spin $+1 / 2$ or heavy holes $(\mathrm{HH})$ of spin $-3 / 2$ in the valence band (VB). From the matrix elements and transition probabilities for allowed transitions [13], it is evident that the probability of heavyhole transitions is three times larger than transitions involving light holes. Thus, the selection rules for the conservation of angular momentum $\left(\Delta m_{j}= \pm 1\right)$ give the relation between the carrier spin $\left(\Pi_{\mathrm{inj}}\right)$, and the degree of circular polarization of the photons $\left(\Pi_{\mathrm{CP}}\right)$ in a bulk semiconductor as

$$
\Pi_{\mathrm{CP}}=\frac{n_{\downarrow}-n_{\uparrow}}{2\left(n_{\downarrow}+n_{\uparrow}\right)}=-\Pi_{\mathrm{inj}} / 2,
$$

where $n_{\uparrow}$ and $n_{\downarrow}$ are the densities of spin-up and spin-down electrons, respectively. In low-dimensional quantum confined systems or biaxially strained semiconductors where the heavy hole-light hole degeneracy is lifted, the relation is reduced to

$$
\Pi_{\mathrm{CP}}=\frac{n_{\downarrow}-n_{\uparrow}}{\left(n_{\downarrow}+n_{\uparrow}\right)}=-\Pi_{\mathrm{inj}} .
$$

In both cases, the photons are emitted with their angular momentum oriented against the direction of spin polarization. The possible optical transitions in a quantum dot for polarized light according to the selection rules are shown in figure 1. It is important to note that the selection rules are valid only when the photon emission is in the direction of the spin polarization of the recombining carriers. In a non-magnetic light source, there is an equal injection of spin-up and spindown carriers which equally pump the left- $\left(\sigma^{-}\right)$and rightcircularly polarized $\left(\sigma^{-}\right)$optical modes. These equal and inphase circularly polarized modes admix to produce a linearly polarized emission. In a spin-polarized light source spin injection is unequal in the two polarization modes, and the selection rules give rise to photon emission in the preferred polarization mode with a net circular polarization. Output

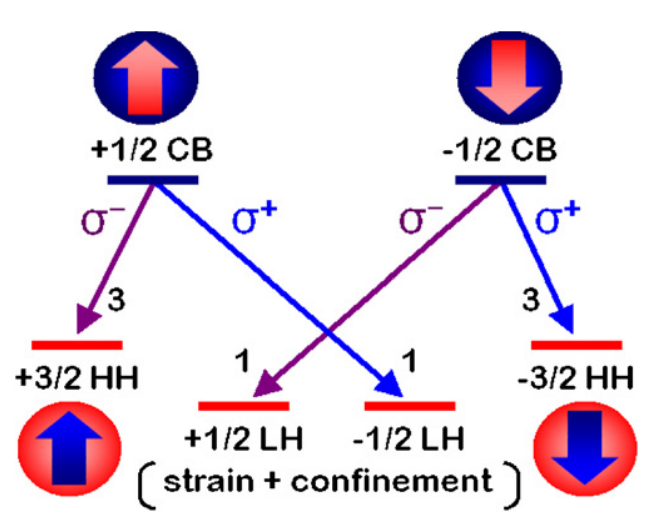

Figure 1. Electric dipole allowed radiative interband transitions and corresponding optical polarization for a QD in which epitaxial strain and quantum confinement have lifted the heavy- and light-hole band degeneracy. From [14].

polarization in accordance with the selection rules has been observed for spin LEDs [7]. These devices are useful for the study of spin injection and spin transport [15]. In spin lasers the output circular polarization can be larger than the spin polarization of the injected carriers, due to the phenomenon of gain anisotropy to be discussed later. Also, lasing occurs with fewer total carriers, than in a randomly polarized laser, driving the preferred polarization mode above the threshold. The resulting reduction in threshold current, as calculated from laser rate equations [8], and from measurements made on quantum well spin VCSELs are shown in figures 2(a) and $(b)$.

\section{Quantum dot spin-polarized VCSELs}

The first electrically injected spin-polarized laser was reported by us. The devices, in the form of a VCSEL, had $\mathrm{In}_{0.2} \mathrm{Ga}_{0.8} \mathrm{As} / \mathrm{GaAs}$ multi-quantum well active regions, and spin-polarized electrons were injected via an epitaxially grown $\mathrm{Fe} / \mathrm{Al}_{0.1} \mathrm{Ga}_{0.9} \mathrm{As}$ Schottky tunnel barrier contact [8]. A maximum threshold current reduction of $11 \%$ and a degree of circular polarization of $23 \%$ were demonstrated at an operating temperature of $50 \mathrm{~K}$. However, for real applications, it is important that the devices are operated at or near room temperature. The electron spin relaxation time $\tau_{\mathrm{s}}$ in these materials is determined by the D'yakanov-Perel (DP) spin scattering process [16]. The value of $\tau_{\mathrm{s}}$ limited by DP scattering in InGaAs quantum wells is $6 \mathrm{ps}$ at room temperature [17], which is much smaller than the radiative lifetime. On the other hand, in quantum dots the near singular density of states and spatial localization of carriers inhibit DP and phonon scattering processes. Thus, a larger value of $\tau_{\mathrm{s}}$ with a weaker temperature dependence is expected and observed [18].

The quantum dot spin VCSEL is schematically shown in figure 3 and is realized by two-step molecular beam epitaxial (MBE) growth and appropriate lithography and contact metallization steps. Details of the growth and fabrication process have been reported elsewhere [19], but is being described here briefly for the convenience of the reader. The InAs quantum dot (QD) density in the active 

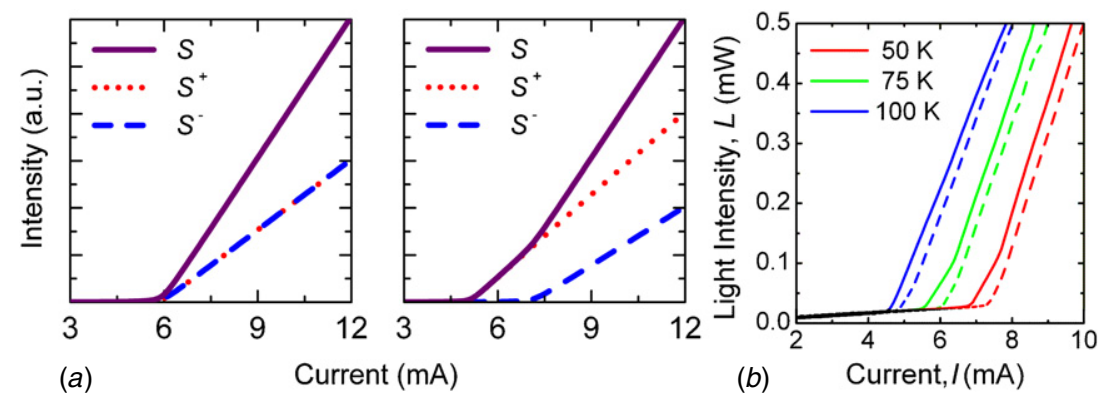

Figure 2. Theoretical light versus current characteristics for a $15 \mu \mathrm{m}$ spin-polarized VCSEL driven with $0 \%$ (left) and $-19.8 \%$ (right) spin-polarized pump currents. The solid line represents the total light intensity, $S$, which is the summation of the right-hand $\left(S^{+}\right.$, dotted line) and left-hand circularly polarized $\left(S^{-}\right.$, dashed line) modes. (b) Light intensity versus current measured at 0 (dashed line) and $5 \mathrm{kOe}$ in the Faraday geometry (solid line). From [8].

(a)

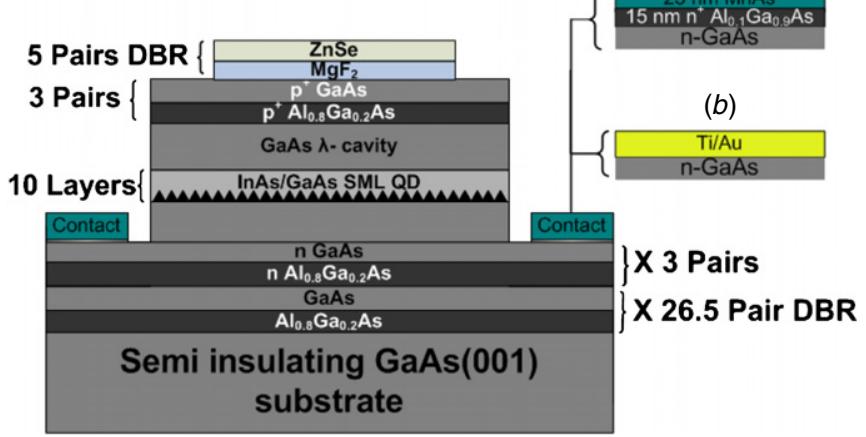

Figure 3. Schematic cross section of an InAs sub monolayer (SML) QD spin VCSEL heterostructure with $(a)$ magnetic and (b) nonmagnetic $n$-contact grown by MBE. From [19].

region is $3 \times 10^{10} \mathrm{~cm}^{-2}$. The device mesa diameters vary from 15 to $30 \mu \mathrm{m}$. To form the spin-polarized electron injector contact, the MnAs $/ \mathrm{n}^{+}-\mathrm{Al}_{0.1} \mathrm{Ga}_{0.9} \mathrm{As}$ Schottky barrier heterostructure is selectively regrown by MBE after a mesa definition. The mesa top surface and side walls are protected during regrowth by an $\sim 2 \mu \mathrm{m}$ thick $\mathrm{SiO}_{2}$ layer which is formed by plasma-enhanced chemical vapor deposition and subsequently patterned to reveal the annular $\mathrm{n}^{+}-\mathrm{GaAs}$ contact layer. After a high temperature annealing for oxide removal from the GaAs surface, $15 \mathrm{~nm} \mathrm{n}^{+}-\mathrm{Al}_{0.1} \mathrm{Ga}_{0.9} \mathrm{As}\left(N_{\mathrm{D}}=\right.$ $10^{19} \mathrm{~cm}^{-3}$ ) and $25 \mathrm{~nm}$ MnAs are epitaxially grown at 630 and $250{ }^{\circ} \mathrm{C}$, respectively. The injection efficiency of the MnAs contacts, as determined from measurements on spin valves, is $50 \%$ and the injected spin polarization is $32 \%$ [20]. Nonmagnetic control VCSELs are also typically fabricated with the same mesa dimensions, but with a Ti/Au bilayer serving as the $n$-contact. The top distributed Bragg reflector (DBR) mirror is formed with the five pairs of $\mathrm{ZnSe} / \mathrm{MgF}_{2}$ deposited by electron beam evaporation. Measurements on the devices mounted in a 7 tesla magneto-optical cryostat are done in the Faraday geometry, for reasons mentioned earlier. Therefore the ferromagnetic contact is polarized perpendicularly to the surface, or along its hard axis. The lasing wavelength of these devices is typically in the range of $0.95-1.0 \mu \mathrm{m}$, depending on the composition, size and heterostructure of the QDs. The degree of circular polarization of the coherent output is measured with the help of a photoelastic modulator, a GlanThompson linear polarizer, a silicon avalanche photodiode with a digital lock-in and low-noise pre-amplifier. The measured output polarization of the QD spin VCSEL at $200 \mathrm{~K}$ and at an injection current of $1.05 I_{\mathrm{th}, 0}$ (where $I_{\mathrm{th}, 0}$ is the threshold current at zero applied magnetic field) is shown in figure $4(a)$. Also shown is the measured out-of-plane magnetization of the MnAs contact, which closely tracks the output polarization. This provides substantive evidence of the injected spin-polarized electrons as the source of the observed output polarization. Furthermore, as shown in figure 4(a), the control VCSEL with non-magnetic contacts exhibits almost zero output polarization. In the past, we have tested other contact configurations to prove the validity of spin injection as the source of the output polarization. These control experiments are extremely important and helpful to eliminate spurious effects as the source of observed polarization.

\section{Threshold current reduction and output polarization}

As mentioned earlier, the output polarization of a spin laser is not related to the injected carrier polarization in accordance with the selection rules. This is because of the nonlinear dynamics and the spin polarization of the gain medium, which gives rise to a large gain anisotropy at biases near threshold. As a result, the output polarization can be much larger than the spin polarization in the active region.

From the laser rate equations, and taking into account the diffusive transport of the injected spin-polarized electrons from the ferromagnetic contact, the following equations have been derived [21] and are being reproduced here. The threshold current $\left(I_{\mathrm{th}}\right)$ is given in terms of threshold photon density $\left(S_{\mathrm{th}}\right)$ by

$$
I_{\mathrm{th}}(H)=q V_{\mathrm{QD}}\left[\frac{1}{\Gamma} \frac{S_{\mathrm{th}}}{\tau_{\mathrm{ph}}}+\frac{B_{\mathrm{sp}} n_{\mathrm{th}}^{2}(1-\beta)}{\left(1+\Pi_{s}(H)\right)^{2}}+\frac{C n_{\mathrm{th}}^{3}}{\left(1+\Pi_{s}(H)\right)^{3}}\right] .
$$

The threshold current reduction can be expressed as

$$
\frac{\Delta I_{\mathrm{th}}(H)}{I_{\mathrm{th}, 0}} \cong \frac{\Pi_{s}\left(\Pi_{s}+2\right)}{\left(1+\Pi_{s}\right)^{2}} .
$$



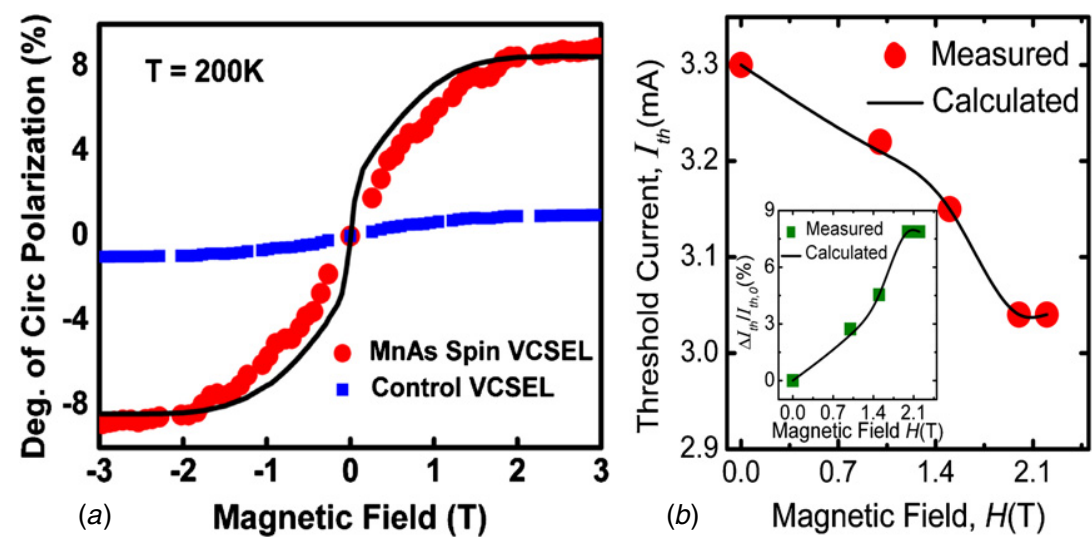

Figure 4. (a) Measured degree of output circular polarization versus magnetic field for a $15 \mu \mathrm{m}$ mesa diameter MnAs spin VCSEL (circle) and control VCSEL (square) measured at $200 \mathrm{~K}$ are shown. The spin VCSEL polarization closely follows the out-of-plane magnetization curve for MnAs (from [19]). (b) Calculated and measured reduction of threshold current with magnetic field applied perpendicularly (hard axis) to the plane of the MnAs contact. The inset shows the calculated and measured percentage reduction of threshold current with field. From [21].
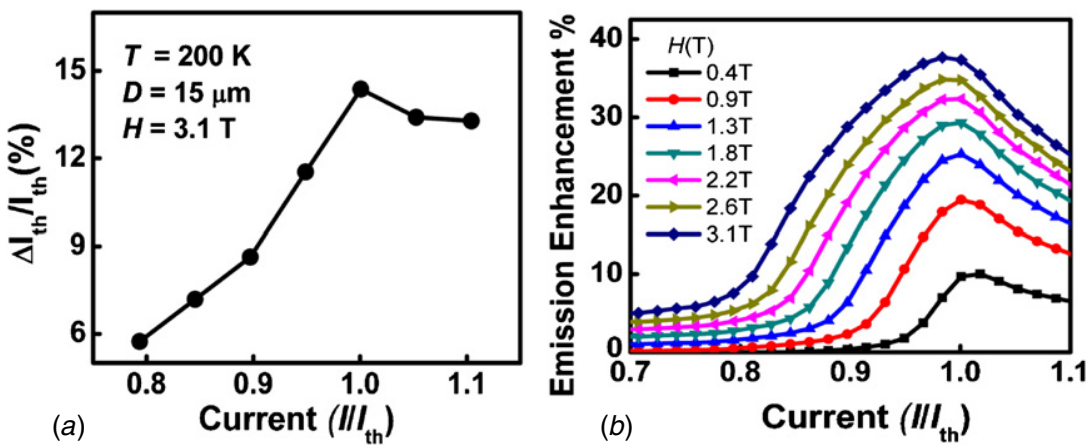

Figure 5. (a) Variation in threshold current reduction $\Delta I_{\mathrm{th}} / I_{\mathrm{th}}$ versus normalized current $\left(I / I_{\mathrm{th}}\right)$ measured at a magnetic field of $3.1 \mathrm{~T}$. (b) Emission intensity enhancement versus normalized current $\left(I / I_{\mathrm{th}}\right)$ measured for different magnetic fields [19].

The output polarization is given by

$\frac{\Pi_{c}\left(H, I_{\mathrm{pump}}\right)}{\Pi_{s}}=-\frac{1+\Gamma v_{g} \tau_{\mathrm{ph}} \frac{\mathrm{d} g}{\mathrm{~d} n} n_{\mathrm{tr}}}{1+\Gamma v_{g} \tau_{\mathrm{ph}} \frac{\mathrm{d} g}{\mathrm{~d} n} n_{\mathrm{tr}}-\Gamma v_{g} \tau_{\mathrm{ph}} \frac{\mathrm{d} g}{\mathrm{~d} n} n_{\mathrm{th}}\left(1-\Pi_{s}\right)}$.

The gain anisotropy near threshold can be expressed as

$$
g_{A}\left(H, I_{\text {pump }}\right)=\frac{g\left(n^{+}\right)}{g\left(n^{-}\right)} \cong \frac{1+\Pi_{s}}{1-\Pi_{s}} .
$$

In these equations $\Pi_{s}$ is the spin polarization in the gain medium, $\beta$ is the spontaneous emission factor, $\beta_{\mathrm{sp}}$ is the radiative recombination coefficient, $C$ is the Auger recombination coefficient, $n_{\text {th }}$ is the threshold carrier density in the cavity, $n_{\mathrm{tr}}$ is the transparency carrier density, $\mathrm{d} g / \mathrm{d} n$ is the differential gain, $\tau_{\mathrm{ph}}$ is the cavity photon lifetime, $q$ is the charge of an electron and $S_{\text {th }}$ is the threshold photon density. The measured and calculated reductions in threshold current are shown in figure $4(b)$. The reduction saturates at $\sim 2$ tesla, providing further evidence of spin injection from the MnAs contact. A spin relaxation time of 150 ps was assumed for the calculations. The other parameters are listed in table 1. The gain anisotropy near threshold results in an emission enhancement. Both the threshold reduction and the
Table 1. Spin laser model parameters and their values for the InAs/GaAs QD spin-VCSEL at $200 \mathrm{~K}$ [21].

\begin{tabular}{lll}
\hline Parameter & Symbol & Value \\
\hline Differential gain & $\mathrm{d} g / \mathrm{d} n$ & $3.4 \times 10^{-14} \mathrm{~cm}^{2}$ \\
Rad. recombination coefficient & $B_{\text {sp }}$ & $9.4 \times 10^{-9} \mathrm{~cm}^{3} \mathrm{~s}^{-1}$ \\
Auger recombination coefficient & $\mathrm{C}$ & $1.5 \times 10^{-27} \mathrm{~cm}^{6} \mathrm{~s}^{-1}$ \\
Carrier capture time & $\tau_{\text {cap }}$ & $45 \mathrm{ps}$ \\
Spin-flip time in barrier & $\tau_{s, b}$ & $300 \mathrm{ps}$ \\
Spin-flip time in QD & $\tau_{s}$ & $150 \mathrm{ps}$ \\
Photon group velocity & $v_{\mathrm{g}}$ & $8.7 \times 10^{9} \mathrm{~cm} \mathrm{~s}^{-1}$ \\
Optical confinement factor & $\Gamma$ & 0.024 \\
Spontaneous emission factor & $\beta$ & $6 \times 10^{-4}$ \\
Photon lifetime & $\tau_{\mathrm{ph}}$ & $1 \mathrm{ps}$ \\
Threshold carrier density & $n_{\mathrm{th}}$ & $4.66 \times 10^{18} \mathrm{~cm}^{-3}$ \\
Transparency carrier density & $n_{\mathrm{tr}}$ & $4 \times 10^{17} \mathrm{~cm}^{-3}$ \\
Ferromagnetic contact polarization & $P_{\text {contact }}$ & 0.31 \\
Spin diffusion length & $\lambda_{\mathrm{sf}}$ & $0.6 \times 10^{-4} \mathrm{~cm}^{-1}$ \\
Barrier volume & $V_{b}$ & $2.1 \times 10^{-11} \mathrm{~cm}^{3}$ \\
Active region volume & $V_{\mathrm{QD}}$ & $2.8 \times 10^{-13} \mathrm{~cm}^{3}$ \\
Charge of an electron & $q$ & $1.6 \times 10^{-19} \mathrm{C}$ \\
\hline
\end{tabular}

emission enhancement go through a maximum near threshold, as shown in figure 5 . 


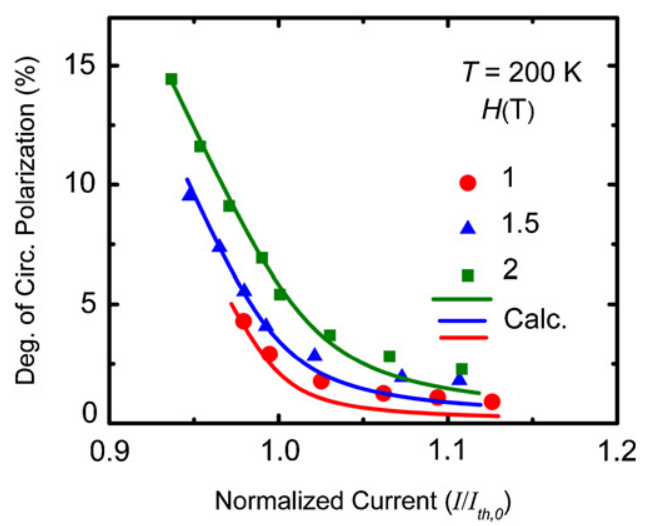

Figure 6. Calculated and measured modulation of output circular polarization of the InAs/GaAs QD spin VCSEL as a function of the normalized pump current at different magnetic fields [21].

\section{Polarization modulation and frequency response of the spin VCSEL}

Electrical injection of spin-polarized carriers in a spin laser provides a convenient means of electrical modulation of the output polarization and amplitude (small-signal frequency response). The calculated and measured variation of the output polarization of a QD spin VCSEL with injected current at $200 \mathrm{~K}$ are shown in figure 6 and they are in reasonably good agreement. Again, the output polarization goes through a maximum near threshold, since the spin polarization of the injected carriers is at a maximum, and rapidly decreases with increasing current since the population of both spin polarizations approaches $n_{\text {th }}$.

The small-signal frequency response of a spin VCSEL has not been measured but can be calculated from the rate equations [22]. Before describing these, it is instructive to briefly review the unique hot carrier dynamics in QDs and QD lasers. From the temperature-dependent measurement of gain in QD lasers [23] and from detailed two- and three-pulse pump-probe measurements [24], it is evident that the QD wetting layer, formed during Stranski-Krastanow growth, has a major influence on laser performance. The localized QD states and the two-dimensional wetting layer/barrier states of the dot heterostructures form an electronically coupled system. Therefore, at room temperature, most of the injected carriers will reside in the wetting layer/barrier states, and equilibrium quasi-Fermi statistics is not valid. Additionally, while injected carriers lose energy and fill the lasing states, carrier heating simultaneously forces them out toward higher energies and leads to their leakage to adjoining barrier layers. Thus, a significant hot carrier density can develop in a QD laser at fairly low injection densities, which leads to severe gain compression at the lasing states and to increased threshold current and reduced small-signal modulation frequency. In fact, the largest $-3 \mathrm{~dB}$ small-signal modulation bandwidth of a $1 \mu \mathrm{m}$ QD laser is only $\sim 6-7 \mathrm{GHz}$ [25], compared to $\sim 25 \mathrm{GHz}$ for an identical $1 \mu \mathrm{m}$ quantum well laser. On the other hand, by incorporating tunnel injection of electrons in the design of the QD laser [24], the above-mentioned hotcarrier related problems can be largely alleviated. In this technique cold electrons are directly injected to the lasing states by tunneling, thereby minimizing heating of the other carriers and phonons and reducing carrier leakage. We have experimentally demonstrated a $3 \mathrm{~dB}$ modulation bandwidth of $23 \mathrm{GHz}$ in a $1 \mu \mathrm{m} \mathrm{In} \operatorname{In}_{0.4} \mathrm{Ga}_{0.6} \mathrm{As} / \mathrm{GaAs}$ tunnel injection QD laser and a substantial reduction of the gain compression factor $\varepsilon$ [24].

The rate equations, which describe the carrier-photon dynamics in a laser, can be modified to include the spin polarization of the carriers in the gain region (QDs) and the circular polarization of the output light. These equations are as follows:

$$
\begin{gathered}
\frac{\mathrm{d} n^{ \pm}}{\mathrm{d} t}=\frac{J^{ \pm}}{q^{d}}-v_{g} g^{ \pm}\left(n^{ \pm}, S^{\mp}\right) S^{\mp}-\frac{n^{+}-n^{-}}{\tau_{s}}-R_{\mathrm{sp}}^{ \pm} \\
\frac{\mathrm{d} S^{ \pm}}{\mathrm{d} t}=\Gamma v_{g} g^{ \pm}\left(n^{ \pm}, S^{\mp}\right) S^{\mp}-\frac{S^{ \pm}}{\tau_{p h}}+\beta \Gamma R_{\mathrm{sp}}^{ \pm},
\end{gathered}
$$

where $n^{ \pm}$are the density of spin-polarized carriers in the active region and $S^{ \pm}$are the density of left- and right-circularly polarized photons produced by the recombination of spinpolarized carriers. $J$ is the injected current density, $v_{g}$ is the photon group velocity, $g$ is the material gain, $R_{\mathrm{sp}}$ is the spontaneous emission rate, $\beta$ is the spontaneous emission factor, $\Gamma$ is the optical confinement factor and $\tau_{\mathrm{ph}}$ is the photon lifetime in the cavity. The polarized carrier densities are expressed as

$$
n^{ \pm}=\frac{n}{2}\left(1 \pm \Pi_{\mathrm{S}}\right)
$$

where $\Pi_{\mathrm{s}}$ is the polarization in the active region. Also $R_{\mathrm{sp}}^{ \pm}=n^{ \pm} / \tau_{r}$, where $\tau_{r}$ is the radiative lifetime of the carriers. The gain of the laser is modeled as

$$
g^{ \pm}=2 g_{0}\left(n^{ \pm}-n_{\text {tr }}\right)\left(1+\varepsilon S^{ \pm}\right)^{-1},
$$

where $g_{0}$ is the differential gain, $n_{\text {tr }}$ is the transparency current density and $\varepsilon$ is the gain compression factor. In a spin-polarized laser near threshold the gain of the favored polarization mode crosses the threshold gain and the device lases with a smaller threshold current $[8,23]$. If the favored mode is $S^{-}$, then $S^{-} \neq 0$ and $S^{+} \cong 0$. It may be noted that in this analysis we neglect the spin polarization of carriers in the barrier regions. It can be shown from the carrier and photon rate equations that the transfer function representing the small-signal modulation response is given by

$$
\frac{\Delta S}{\Delta J}=\frac{\Gamma\left(c+\frac{\beta}{\tau_{r}}\right) \frac{1}{q d}}{\left[\mathrm{j} \omega+\left(\frac{1}{\tau_{\mathrm{ph}}}-b \Gamma\right)\right]\left[\mathrm{j} \omega+\left(c+\frac{1}{\tau_{r}}\right)\right]+\Gamma b\left(c+\frac{\beta}{\tau_{r}}\right)}
$$

with $b=2 v_{g} g_{0}\left(\frac{a \bar{n}}{2}-n_{\text {tr }}\right)(1-2 \varepsilon \Delta S), c=2 v_{g} g_{0} \frac{a}{2} \bar{S}^{-}(1-$ $\left.\varepsilon \bar{S}^{-}\right)$and $a=1+\Pi_{S}$. Here $d$ is the total height of the active region. Also $\bar{n}$ and $\bar{S}$ denote steady state (dc) values of the parameters. Finally, the resonance frequency of the smallsignal response for the favored $S^{-}$mode can be expressed as

$$
\omega_{r}^{2} \cong \frac{2 v_{g} g_{0} \bar{S}^{-}}{\tau_{\mathrm{ph}}}\left[\left(\frac{1+\Pi_{\mathrm{S}}}{2}\right)\left(1-\varepsilon \bar{S}^{-}\right)\right] .
$$

Figures 7(a) and (b) show the calculated smallsignal modulation response at different values of the spinpolarization for two values of the gain compression factor $\varepsilon$. 


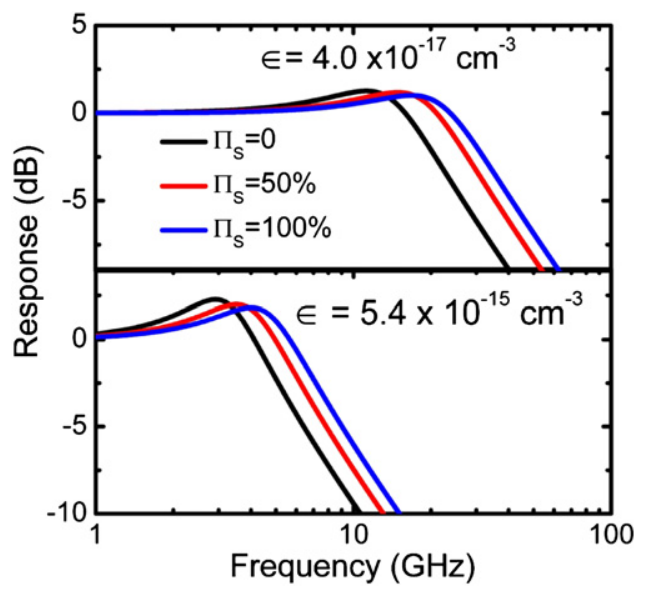

Figure 7. Calculated small signal modulation bandwidth of a $1 \mu \mathrm{m}$ spin laser showing the effect of hot carriers and associated gain compression. $(a)$ and $(b)$ correspond to a tunnel injection and a conventional separate confinement heterostructure $(\mathrm{SCH})$ laser design, respectively.

Specifically, (a) and (b) correspond to the cases of a tunnel injection QD laser and a conventional QD laser, respectively. The parameter values listed in table 1 for $1.1 \mu \mathrm{m}$ QD lasers have been used in the calculations. Thus, for unpolarized lasers, the $3 \mathrm{~dB}$ bandwidth, $f_{-3 \mathrm{~dB}}$ is $\sim 23 \mathrm{GHz}$ (a) and $\sim 6 \mathrm{GHz}$ (b), which is exactly the same as the measured values mentioned earlier for tunnel injection and conventional QD lasers, respectively. It is also evident that in both cases, the modulation bandwidth steadily increases with increasing spin polarization of the injected carriers. The increase is a maximum at or very near threshold (of the favored mode) and can be understood considering the reduction in threshold or the increase in gain (gain anisotropy) [21] of the favored mode.

\section{Quantum dot spin-polarized single photon source}

In self-organized QDs, there is optical in-plane anisotropy of the dot transitions, and the confinement potential does not possess axial symmetry. This is particularly true when the epitaxial growth conditions are fixed by very low areal density of the dots. Due to electron-hole anisotropic exchange interaction and lowering of the confinement symmetry from $\mathrm{D}_{2 \mathrm{D}}$, mixed excitonic eigenstates with finite splitting are formed [26, 27] and as a result linearly polarized emission along the axis of anisotropy can be observed. It has also been reported that the effects of the anisotropic exchange interaction can be reduced or eliminated by the application of in-plane [28] or out-of-plane [29] magnetic fields or electric fields $[30,31]$. It is therefore of interest to study the output polarization characteristics of an electrically injected spinpolarized single-photon source as a function of the magnetic field, for applications in quantum cryptography, exciton-based spin memories and for developing an understanding of the electronic properties of self-organized quantum dots with asymmetry in the dot geometry resulting from growth related phenomena. The device used is very similar to that reported by

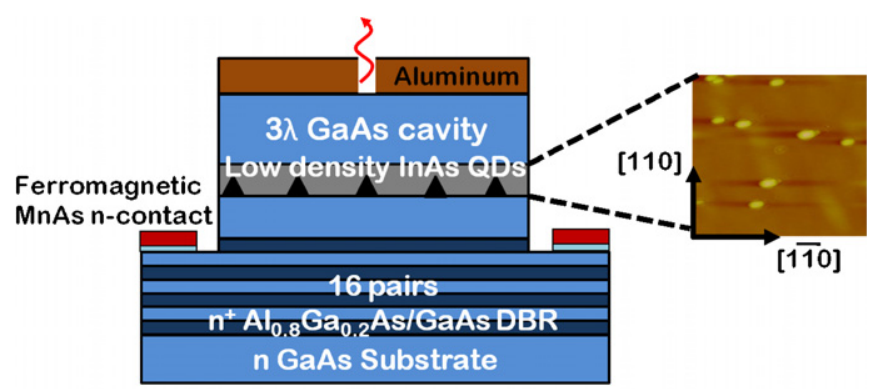

(a)

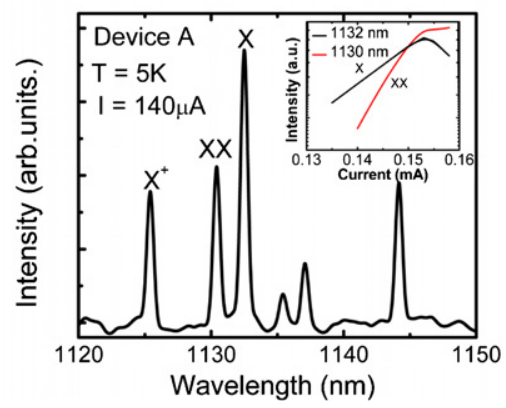

(b)

Figure 8. (a) Fabricated device (color online) with the ferromagnetic $\mathrm{MnAs} / \mathrm{Al}_{0.1} \mathrm{Ga}_{0.9}$ As tunnel injector contact. The AFM image of a layer of quantum dots is shown in the inset; (b) electroluminescence spectrum of the device measured with an injection current of $140 \mu \mathrm{A}$ showing exciton, biexciton and charged exciton emission from a single QD. The linear and quadratic variations of different components (shown in the inset) in the electroluminescence spectra confirm the presence of excitonic and bi-excitonic transitions [9].

Bennett et al [32], except for the MnAs Schottky tunnel contact to inject spin-polarized electrons which recombine with holes in the quantum dots. The device heterostructure, grown by one-step MBE and schematically shown in figure 8(a), consists of an n-doped 16-period $\mathrm{GaAs} / \mathrm{Al}_{0.8} \mathrm{Ga}_{0.2} \mathrm{As}$ DBR mirror, a $3 \lambda$ GaAs cavity with a single layer of very low density $\left(\sim 10^{8} \mathrm{~cm}^{-2}\right)$ InAs/GaAs QDs positioned one wavelength from the Bragg mirror and a $75 \mathrm{~nm} \mathrm{Al}$ layer on top. From atomic force microscope (AFM) imaging it is apparent that the dots are slightly elongated along the $\left[\begin{array}{ll}1 \overline{1} & 0\end{array}\right]$ in-plane crystallographic direction. The mesa-shaped device is fabricated by standard wet and dry etching, photolithography and metallization techniques. The device mesa diameters vary from 10 to $40 \mu \mathrm{m}$. To form the spin-polarized electron injector contact, an $\mathrm{MnAs} / \mathrm{n}^{+}-\mathrm{Al}_{0.1} \mathrm{Ga}_{0.9} \mathrm{As}$ Schottky barrier heterostructure is selectively regrown by MBE after the mesa definition. The top Al layer serves several purposes: it is used as a non-alloyed p-ohmic contact; a micron-sized aperture is formed in this layer by electron beam lithography and etching to isolate and transmit the emission from a single dot underneath it; the emission from other dots located elsewhere is reflected back with a near $-\pi$ phase change and partly absorbed, at the emission wavelength, which ensures that the only photons being emitted from the device are from the single dot below the aperture. The devices exhibit diode-like behavior with a forward turn-on voltage of $\sim 2.5 \mathrm{~V}$; the high turn-on and series resistance of ohms can be attributed to the unannealed magnetic n-contact. 


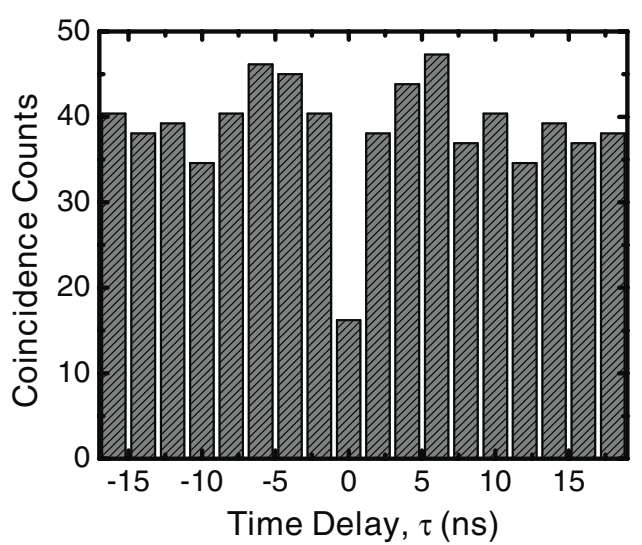

Figure 9. Coincidence counts versus delay time histogram measured at the bi-excitonic peak of $1130 \mathrm{~nm}$ by the

Hanbury-Brown and Twiss arrangement in the unfiltered configuration. The coincidences are proportional to the second-order correlation function of the source, $g^{2}(\tau)$. The zero delay peak area is suppressed to $34 \%$ of the area of the other peaks, indicating a three-fold decrease in multiphoton emission relative to a Poissonian source of equal intensity [9]

Electroluminescence spectra measured at $5 \mathrm{~K}$ show distinct exciton and bi-exciton transitions, as shown in figure $8(b)$. The linewidth of the observed transitions is $\sim 0.1 \mathrm{~nm}$. Single photon emission from the devices is investigated by measuring the normalized second-order correlation function $g^{2}(\tau)=\langle I(t) I(t+\tau)\rangle /\langle I(t)\rangle\langle I(t+\tau)\rangle$, where $I(t)$ is the electroluminescence intensity at time $t$. Measurements were made at $5 \mathrm{~K}$ with a Hanbury-Brown and Twiss experimental arrangement consisting of a 50/50 beamsplitter and two identical photon counting avalanche photodiodes. The time resolution of the system is $\sim 10 \mathrm{ps}$. The histogram shown in figure 9 is recorded under $\mathrm{CW}$ electrical excitation at the bi-exciton emission and is proportional to the second-order correlation function in the low count rate limit. No selection of output polarization was made in these measurements. The dip in the correlation signal at zero time delay, which is referred to as antibunching, is the evidence of the strong suppression of multiphoton emission. The value of $g^{2}(0)$ is 0.45 . We have also made identical measurements for the exciton emission, for which $g^{2}(0)$ is 0.34 . The data indicate that after a single photon emission, there is a finite time delay before the QD captures an electron and a hole and can re-emit a photon.

The output polarization characteristics of the biexciton photons are measured in the Faraday geometry by mounting them in a magneto-optical cryostat. The biexcitonic wavelength was chosen so as to take the advantage of the higher emission intensity. The degree of linear polarization (DOLP) of the emission, $\left(\pi_{x}-\pi_{y}\right) /\left(\pi_{x}+\pi_{y}\right)$, is determined by passing the output through a Glan-Thompson linear polarizer, with its axis along the [1 000$]$ or [1 110$]$ crystallographic directions. The degree of circular polarization (DOCP), $\left(\sigma^{+}-\sigma^{-}\right) /\left(\sigma^{+}+\sigma\right)$, is analyzed using a photoelastic modulator operating at $50 \mathrm{KHz}$ and a Glan-Thompson linear polarizer. The measured linear and circular polarizations are shown in figures $10(a)$ and $(b)$. It may be noted here that the polarization of an ensemble of photons is being measured here. From the data in figures $10(a)$ and $(b)$ it is evident that the output circular polarization has a zero or very low value up to a magnetic field of $\sim 0.9$ tesla beyond which it quickly increases to a saturation value. The linear polarization shows an almost complementary trend. It is important to note that the output circular polarization does not follow the magnetization of the MnAs contact, as in the case of a spin VCSEL. Measurements were also made on an identical device, but with the ferromagnetic MnAs contact replaced by a non-magnetic $\mathrm{Ni} / \mathrm{Ge} / \mathrm{Au} / \mathrm{Ti} / \mathrm{Au}$ alloyed contact. The output polarization characteristics of this device are shown in figure $10(c)$. The value of circular polarization is negligible at all values of applied magnetic field, while the linear polarization exhibits the same behavior as the device with ferromagnetic contact.

These experimental observations can be explained by invoking the exciton fine structure in the QD and the effects of electron-hole exchange splitting, due to in-plane quantum dot rotational asymmetry, and the effect of Zeeman splitting on the spin eigenstates and their coupling to the photon field. It may be remembered that in the Faraday geometry the magnetization of the MnAs contact of the single-photon source is along the hard axis. Therefore for zero applied magnetic field, the recombination of electrons and holes with zero net spin polarization will produce photons which will be predominantly linearly polarized due to transitions from the degenerate biexciton state to the excitonic ground state involving mixed spin eigenstates. With the application of a magnetic field, spin-polarized electrons are injected by the MnAs Schottky tunnel contact, and it is expected that the photons emitted would have some degree of net circular polarization, increasing with increasing magnetic field as in the case of spin lasers. However the strong exchange interaction due to the in-plane anisotropy of the quantum dot ensures that the spin eigenstates are mixed states and are coupled to a photon field which is linearly polarized [29, 33]. The measured circular polarization remains zero until a field of $B \sim 1$ tesla is reached, when the Zeeman splitting becomes comparable to the bright states fine structure splitting $\delta_{1}$ and this results in pure spin eigenstates. Hence the recombination of spin-polarized electrons now produces circularly polarized photons, with the degree of circular polarization dictated by the magnetization of the contact, its injection efficiency and the transport characteristics of the spin-polarized electrons from the MnAs contact to the active quantum dot. Comparable values of the Zeeman splitting and $\delta_{1}$ at $\mathrm{B} \sim 1 \mathrm{~T}$ have also been reported [32] for $\mathrm{In}_{0.4} \mathrm{Ga}_{0.6}$ As quantum dots. Coincidentally, the magnetization of the MnAs layer along the hard axis also exhibits saturation at $B \sim 1$ tesla. This gives rise to the abrupt switching behavior of the measured circular polarization. A more detailed analysis of our results suggests that the $g$-factor required to compensate an exchange splitting $\delta_{1}$ of $\sim 480 \mu \mathrm{eV}$ for a magnetic field of 1 tesla is $\sim 6$, which is approximately six times the typical values of $g_{\|}$in InGaAs QDs. The large $g$ factor can only be attributed to the Boltzmann distribution (kT at $5 \mathrm{~K}-430 \mu \mathrm{eV}$ ) which also contributes to the spin splitting in addition to the Zeeman interaction. It may be noted that the 

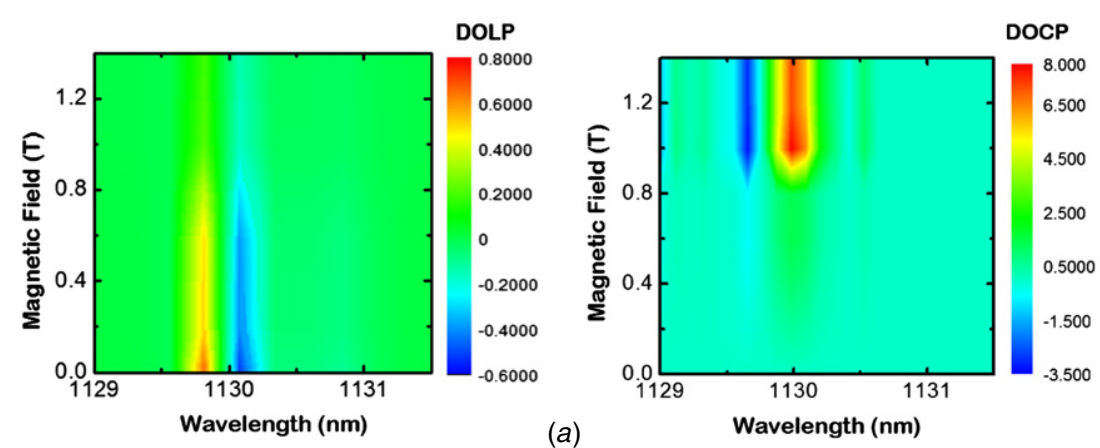

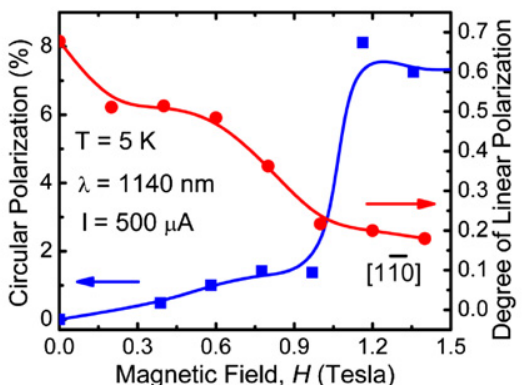

(b)

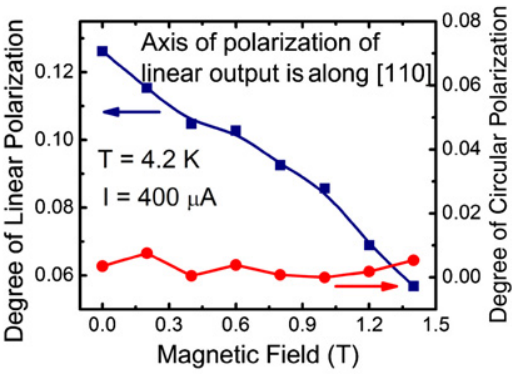

(c)

Figure 10. (a) Variation of linear polarization $\left(\pi_{x}-\pi_{y}\right) /\left(\pi_{x}+\pi_{y}\right)$ in the direction of the QD axis of anisotropy and output circular polarization $\left(\sigma^{+}-\sigma^{-}\right) /\left(\sigma^{+}+\sigma^{-}\right)$versus magnetic field in the Faraday geometry at $5 \mathrm{~K}$ measured around the biexciton emission wavelength region. The magnetic field-induced splitting into quadruplet states is apparent in the circularly polarized electroluminescence spectrum for $B \| z \sim 0.9$ tesla. (b) Net output circular polarization versus magnetic field measured in the Faraday geometry. (c) Polarization characteristics of the device with the bi-excitonic emission peak at $1100 \mathrm{~nm}$ and non-magnetic contacts are shown. Also shown in $(b)$ and (c) is the variation of linear polarization in the direction of the QD axis of anisotropy versus magnetic field in the Faraday geometry [9].

output polarization behavior of the device with non-magnetic contacts (figure $10(c)$ ), with zero circular polarization up to fields of $\sim 2$ tesla and a linear polarization variation similar to the magnetic device confirms the varying nature of the spin eigenstates of the QD.

The observed polarization behavior is ultimately determined by the growth kinetics and anisotropies of the growth surface during the epitaxy of the QDs. As the dot density is decreased, the anisotropy along the [1 10$]$ direction becomes more pronounced. It has been suggested that asymmetries in the underlying layers can also give rise to anomalous polarization characteristics. The device described here, if operated at higher temperatures, would be useful for polarization-encoded cryptography where the emitted photons would have a preferred polarization. This is possible with GaN-based heterostructures.

\section{Conclusion}

Realization of quantum dot spin-polarized light sources requires a multi-disciplinary approach involving physics, material science and micro-fabrication engineering for their physical implementation. Future work will include the optimization of high-temperature performance and the incorporation of efficient spin injectors for device operation at room temperature in the absence of external magnetic fields. Prediction of long spin relaxation time in nitride-based III-V semiconductors [34], which promise high temperature operation, has triggered substantial efforts toward optimizing the epitaxy and overcoming fundamental hurdles associated with spin injection, transport and detection. Although formidable challenges still remain rapid strides have been made over the past few years.

\section{References}

[1] Sato I, Yamashima R, Kadowaki K, Yamamoto J, Shibata T and Soai K 2001 Asymmetric induction by helical hydrocarbons: [6]- and [5]helicenes Angew. Chem. Int. Ed. 401096

[2] Chang-Hasnain C J, Harbison J P, Hasnain G,

Von Lehmen A C, Florez L T and Stoffel N G 1991

Dynamic, polarization, and transverse mode characteristics of vertical cavity surface emitting lasers IEEE J. Quantum Electron. 271402

[3] Shimuzi M, Mukaihara T, Koyama F and Iga K 1991 Polarization control for surface emitting lasers Electron. Lett. 271067

[4] Robert I, Moreau E, Gérard J M and Abram I 2001 Towards a single-mode single photon source based on single quantum dots J. Lumin. 94-95 797-803

[5] Moreau E, Robert I, Gérard J M, Abram I, Manin L and Thierry-Mieg V 2001 Single-mode solid-state single photon source based on isolated quantum dots in pillar microcavities Appl. Phys. Lett. 792865

[6] Rakher M T, Stoltz N G, Coldren L A, Petroff P M and Bouwmeester D 2008 Polarization-switchable single photon source using the Stark effect Appl. Phys. Lett. 93091118

[7] Fiederling R, Keim M, Reuscher G, Ossau W, Schmidt G, Waag A and Molenkamp L W 1999 Injection and detection of a spin-polarized current in a light-emitting diode Nature $402787-90$ 
[8] Holub M, Shin J, Saha D and Bhattacharya P 2007 Electrical spin injection and threshold reduction in a semiconductor laser Phys. Rev. Lett. 98146603

[9] Bhattacharya P, Das A, Basu D, Guo W and Heo J 2010 An electrically injected quantum dot spin polarized single photon source Appl. Phys. Lett. 96101105

[10] Benson O, Santori C, Pelton M and Yamamoto Y 2000 Regulated and entangled photons from a single quantum dot Phys. Rev. Lett. 842513

[11] Ramon G, Mizrahi U, Akopian N, Braitbart S, Gershoni D, Reinecke T L, Gerardot B D and Petroff P M 2006 Emission characteristics of quantum dots in planar microcavities Phys. Rev. B 73205330

[12] Rudolph J, Hägele D, Gibbs H M, Khitrova G and Oestreich M 2003 Laser threshold reduction in a spintronic device Appl. Phys. Lett. 824516

[13] F Meier and B P Zakharchenya 1984 Optical Orientation (Amsterdam: Elsevier)

[14] Holub M and Bhattacharya P 2007 Spin-polarized light-emitting diodes and lasers J. Phys. D: Appl. Phys. 40 R179

[15] Kioseoglou G, Hanbicki A T, Sullivan J M, van 't Erve O M J, Li C H, Erwin S C, Mallory R, Yasar M, Petrou A and Jonker Berend T 2004 Electrical spin injection from an n-type ferromagnetic semiconductor into a III-V device heterostructure Nat. Mater. 3799

[16] D'yakonov M I and Perel' V I 1971 Fiz. Tverd. Tela 133581 D'yakonov M I and Perel' V I 1971 Sov. Phys. Solid State 13 3023

[17] Ohno Y, Terauchi R, Adachi T, Matsukura F and Ohno H 2000 Electron spin relaxation beyond D'yakonov-Perel' interaction in GaAs/AlGaAs quantum wells Physica E 6817

[18] Tackeuchi1,2 A, Ohtsubo3 R, Yamaguchi3 K, Murayama1 M, Kitamura1 T, Kuroda1 T and Takagahara4 T 2004 Spin relaxation dynamics in highly uniform InAs quantum dots Appl. Phys. Lett. 843576

[19] Basu D, Saha D, Wu C C, Holub M, Mi Z and Bhattacharya P 2008 Electrically injected InAs/GaAs quantum dot spin laser operating at $200 \mathrm{~K}$ Appl. Phys. Lett. 92091119

[20] Saha D, Holub M, Bhattacharya P and Liao Y C 2006 Epitaxially grown $\mathrm{MnAs} / \mathrm{GaAs}$ lateral spin valves $\mathrm{Appl}$. Phys. Lett. 89142504

[21] Basu D, Saha D and Bhattacharya P 2009 Optical polarization modulation and gain anisotropy in an electrically injected spin laser Phys. Rev. Lett. 102093904
[22] Lau K Y, Bar-Chaim N, Ury I, Harder Ch and Yariv A 1983 Direct amplitude modulation of short-cavity GaAs lasers up to X-band frequencies Appl. Phys. Lett. 431

[23] Matthews D R, Summers H D, Smowton P M and Hopkinson M 2002 Experimental investigation of the effect of wetting-layer states on the gain-current characteristic of quantum dot lasers Appl. Phys. Lett. 814904

[24] Bhattacharya P, Ghosh S, Pradhan S, Singh J, Wu Zong-Kwei, Urayama J, Kim K and Norris T B 2003 Carrier dynamics and high-speed modulation properties of tunnel injection InGaAs-GaAs quantum-dot lasers IEEE J. Quantum Electron. 39952

[25] Kamath K, Phillips J, Jiang H, Singh J and Bhattacharya P 1997 Small-signal modulation and differential gain of single-mode self-organized $\operatorname{In}_{0.4} \mathrm{Ga}_{0.6} \mathrm{As} / \mathrm{GaAs}$ quantum dot lasers Appl. Phys. Lett. 702952

[26] Koudinov A V, Akimov I A, Kusrayev Yu G and Henneberger F 2004 Optical and magnetic anisotropies of the hole states in Stranski-Krastanov quantum dots Phys. Rev. B 70 241305(R)

[27] Bayer $\mathrm{M}$ et al 2002 Fine structure of neutral and charged excitons in self-assembled $\operatorname{In}(\mathrm{Ga}) \mathrm{As} /(\mathrm{Al}) \mathrm{GaAs}$ quantum dots Phys. Rev. B 65195315

[28] Stevenson R M, Young R J, Atkinson P, Cooper K, Ritchie D A and Shields A J 2006 A semiconductor source of triggered entangled photon pairs Nature 439179

[29] Krizhanovskii D N, Ebbens A, Tartakovskii A I, Pulizzi F, Wright T, Skolnick M S and Hopkinson M 2005 Individual neutral and charged $\mathrm{In}_{x} \mathrm{Ga}_{1-x}$ As-GaAs quantum dots with strong in-plane optical anisotropy Phys. Rev. B 72161312

[30] Gerardot B D et al 2007 Manipulating exciton fine structure in quantum dots with a lateral electric field Appl. Phys. Lett. 90041101

[31] Kowalik K, Krebs O, Lemaîre A, Laurent S, Senellart P, Voisin P and Gaj J A 2005 Influence of an in-plane electric field on exciton fine structure in InAs-GaAs self-assembled quantum dots Appl. Phys. Lett. 86041907

[32] Bennett A J, Unitt D C, See P, Shields A J, Atkinson P, Cooper K and Ritchie D A 2005 Microcavity single-photon-emitting diode Appl. Phys. Lett. 86181102

[33] Bayer M 2003 Exciton complexes in self-assembled In(Ga)As/GaAs quantum dots Single Quantum Dots vol 90 ed Claus E Ascheron and Hans J Koelsch (Berlin: Springer) p 93

[34] Dietl T, Ohno H and Matsukura F 2001 Hole-mediated ferromagnetism in tetrahedrally coordinated semiconductors Phys. Rev. B 63195205 